\title{
Photoplethysmography Based Remote Health Monitoring System
}

\author{
Malek A. Harbawi ${ }^{1}$, Muhammad I. Ibrahimy ${ }^{2}$, S. M. A. Motakabber ${ }^{3}$ \\ Electrical and Computer Engineering, Kulliyyah of Engineering, International Islamic University Malaysia \\ 1'malyk19@yahoo.com \\ 2ibrahimy@iium.edu.my \\ 3amotakabber@iium.edu.my
}

\begin{abstract}
One of the world's most leading killer diseases is the cardiovascular disease, which accounts for 16.7 million deaths annually. Out of the total population in the world, about 22 million people run the risk of sudden heart failure. However, saving the lives of cardiac patients can be improved by the emergency monitoring so that the initiation of treatment can be taken up within the crucial hour. The acquired signals by pulse oximetry provide significant information about the heart-rate, arterial blood oxygenation, blood pressure and respiratory-rate. Telemedicine provides a great impact in the emergency monitoring of patients located in remote nonclinical environments. A home cardiac telemedicine emergency system based on photoplethysmography has been developed. The acquired signals are processed, transmitted and stored in a local PC. Finally, the data are sent to the remote terminal located at the hospital through internet. The diagnoses are done by specialists from the reading and action can be immediately taken in emergency cases.
\end{abstract}

Keywords-pulse oximeter; heart rate; blood oxygenation; photoplethysmograph; motion artifact; telemedicine; wearable sensors

\section{INTRODUCTION}

There has been an increasing demand for a complete wearable monitoring system for the telemedicine purpose. An online, continuous, and real-time monitoring allows not only the detection of abrupt change of the patient's health, but also the assessment of the right dose and timing of medication. This type of monitoring has become a highly demented for the daily life of a wide slot of people, especially elderly patients who need continuous monitoring around the clock. Subsequently, allowing such patients to engage their normal activities of the daily life, rather than staying at home or close to specialized medical services, which in turns may improve the quality of life. However, moving the focus of care from the hospital to the ambulatory environment can bring about considerable economic benefits. On the other hand, relying on the traditional health examinations is not enough, especially for cardiac patients. In this regard, it is commonly known that cardiac problems may disappear during the examination time or even when the patient is hospitalized. In addition, continuous monitoring is a very useful tool in some places like bathroom, which is one of the most dangerous places in the home. According to [1], more than 10,000 people mostly hypertensive and die in bathrooms every year. As an answer for the previous demands, there have been many attempts to develop a reliable ambulatory monitoring system to meet the previous requirements. However, the primary designs were faced mostly by the bulky size, wearer discomfort, and low signal quality. In addition, the power consumption was also a serious issue. Moreover, there has been an argument about the best sensor architecture and place that would provide both wearer comfort and acquisition stability. For instance, people tend to remove any ambulatory device that might discomfort them, especially during the shower, even if it was a wrist-watch. Consequently, it is necessary to address the wearable sensor shape among the other issues. Regardless hardware design issues, software algorithms have been extensively studied. The main obstacle with the software is the motion artifacts, which are induced by wearer motion. And since the system is designed to be wearable, then the motion is an inevitable case, so it must be tackled without restricting the wearer motion.

Wearable sensors are implemented using different monitoring techniques. These techniques started with the wearable ECG sensor [2] till the most recent researches on the textile sensors [3]. Pulse oximetry (PO) or photoplethysmograph (PPG) sensor is a very useful technique for monitoring both heart rate and blood oxygenation noninvasively [4]. This sensor normally has two LEDs in different ranges, namely red and infrared, and one photo detector. POs have a variety of commercial shapes and brands, but they have the same concept, that is utilizing the pulsation nature of arterial blood to get an impression about the cardio-pulmonary system functions. PO sensors can be attached to the fingertip, ear lobe, forehead, toes, etc. but the theoretical ideal place for it is the fingertip [5]. The concept of PO relies on emitting two light beams on an extremity of an organ in the range of red and infrared one following the other, and then detecting either the transmitted (transmissive mode) or the reflected (reflective mode) light from the extremity by a photo detector. Light beams are affected mainly by reflection, scattering, absorption and diffusion on and through the extremity tissues and bones. By exploiting absorption properties of the extremity, which is varying according to the blood pumping (heart-rate) and blood's color (oxygen saturation), PPG signal and oxygen saturation can be obtained. Choosing two different light wavelengths in the red and infrared comes due to the fact that oxygenated hemoglobin $\left(\mathrm{HbO}_{2}\right)$ and deoxygenated hemoglobin $\mathrm{Hb}$ have distinguishable absorption criteria in these ranges, which allows the quantification of their amounts and hence the oxygen saturation. 
PO has been widely employed in the biomedical field. Its usage ranges from the battlefield to home as a monitoring device. It gains its advantages due to its invasivity, versatility, and low-cost. Among the previous pros, it shows a high sensitivity to the motion artifacts and ambient light, which limits its applicability, especially in the ambulatory field. Moreover, considering such device as a wearable sensor deteriorates its accuracy and complicates the artifacts' distortion. Hence, to accommodate with the accuracy and fidelity measurements, this study addresses the wearable PO sensor in terms of the suitable configuration, acquiring place, size, weight, power consumption, and motion artifact reduction. However, the main focus is guided to the development of a robust algorithm to deal with motion artifact distortion.

\section{OPtICAL PROPERTIES OF HUMAN TISSUES}

Most of human tissues is considered an opaque or a semitransparent medium owing to the presence of microscopic entities, including macromolecules, cells, water pools, layer structures, and light-absorbing substances [6] [7]. Light propagates in this heterogeneous structure under the effect of four factors, namely reflection, refraction, scattering and absorption [8]. The measurable effects of light-tissue interaction are utilized in the biomedical field. When the light propagates in the biological tissues, the influence of the blood cycle on the light pathway allows the quantification of viable information about cardio-pulmonary system [9]. Light pathway is being mainly changed by the arterial blood pulsation which causes an oscillation synchronized with this pulsation, as result there is a modulation of the propagated light. In addition, blood's chromospheres, which give the blood its distinct color, affect the light penetration through the tissue since light absorption of a colored substance depends on the wavelength of the penetrated light. Exploiting such properties has been extensively used in the biomedical devices. Similarly the PPG measures light intensity changes during penetration into tissue, which led to the invention of the PO that provides non-invasive arterial blood oxygenation measurement. The arterial blood is mainly a composite of colored hemoglobin derivatives of oxyhemoglobin $\left(\mathrm{HbO}_{2}\right), \quad$ deoxyhemoglobin $(\mathrm{Hb})$ carboxyhemoglobin $(\mathrm{CHb})$ and methemoglobin (metHb). Each of these derivatives has its own absorption characteristics, but only one of them can bind the oxygen, namely $\mathrm{HbO}_{2}$. According to the distinguishable absorption criteria of these hemoglobin derivatives, the quantifying the $\mathrm{HbO}_{2}$ is possible when four light beams in four distinct ranges of wavelength are emitted on a certain extremity surface of the human body. Nevertheless, an approximation scenario in the optical widow range may allow considering only two main species, namely $\mathrm{HbO}_{2}$ and $\mathrm{Hb}[10]$, thus it facilitates the calculation process. The absorption characteristics of $\mathrm{HbO}_{2}$ and $\mathrm{Hb}$ have been widely investigated, especially in the optical window of human tissues. This distinguishable absorption is shown in Fig. 1.

\section{METHODOLOGY}

The methodology in this research is divided into two parts, namely sensor hardware design issue and the programming software which run the process. In addition, it includes the wireless transmission that is used. Figure 2 shows the flowchart of the methodology steps.

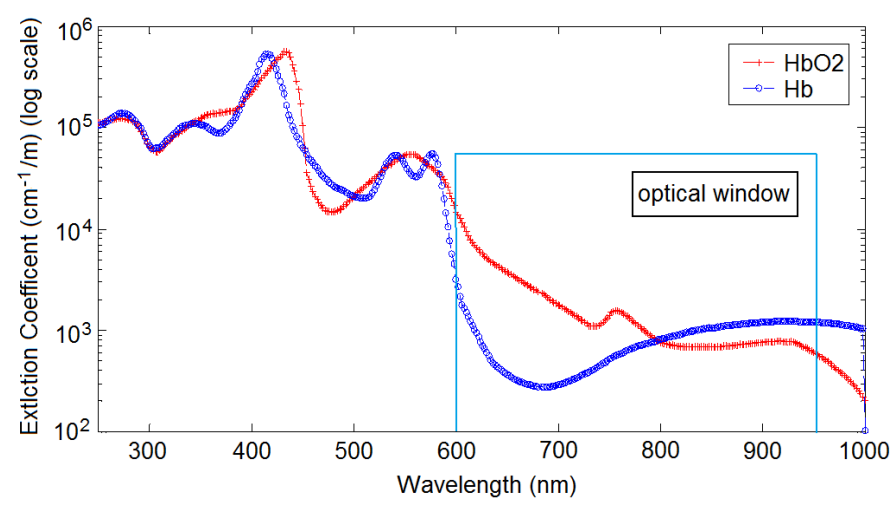

Fig. 1. Absorption characteristics of $\mathrm{HbO}_{2}$ and $\mathrm{Hb}$ for typical human blood

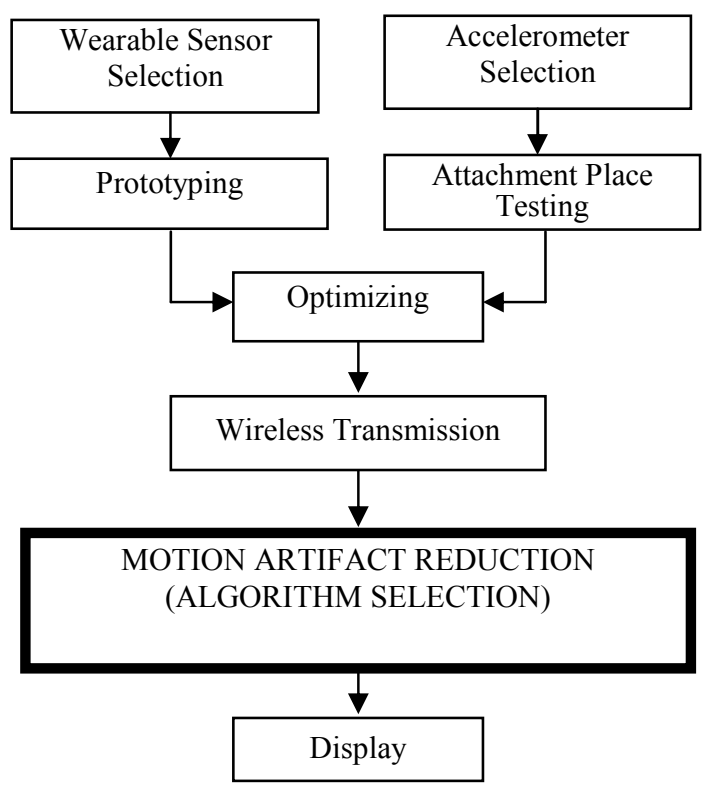

Fig. 2. Flow chart describes the methodology steps

Firstly, the shape and the placement of the sensor are chosen and tested in parallel with the motion accelerometer in order to get motion reference signal. The sensor includes acquisition elements (LEDs and photodiode), microprocessor, and wireless transmitter and small battery as power supply [11]. The design takes into the consideration of components' size, weight and power consumption as well as the comfort of the patient. In this research, the focus is guided into the ringshape wearable PO [10]. This design has the advantage of its hardware architecture, in which it can be worn anytime and anywhere. The sensor is divided into two parts, namely the acquisition part (outer surface of the inner face) and the housing part (inside the core). Such design keeps minimizing the effect of patient's motion, ambient light and skin pressure by keeping the share part isolated from the inner-ring. The signal acquired by the sensor is sent to a local PC which is connected to the internet. This signal is processed by the variable step-size (VSS) least mean square (LMS) adaptive 
filter in the PC. The choice of VSS is coming due to the improvement that it gives over the constant step-size, in terms of the convergent-time and the accuracy. A complete implementation of the adaptive filter is shown in Fig. 3.

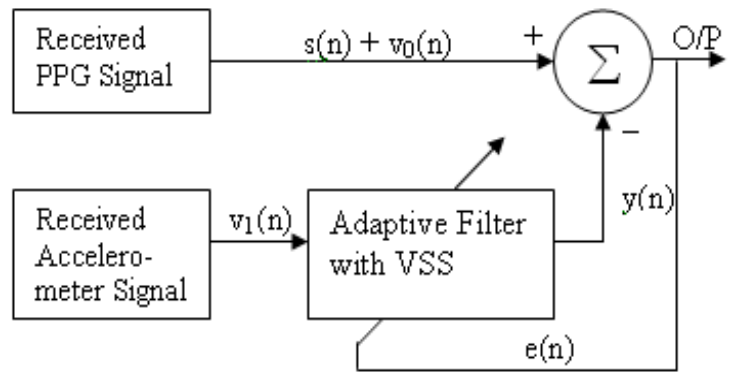

Fig. 3. The proposed adaptive filtering technique

The step-size of the developed VSS algorithm for ANC, is described as follows:

From Fig. 3, the general equation which is describing the LMS is given by:

$$
y(n)=X^{T}(n) W(n)
$$

Minimizing the error $\mathrm{e}(\mathrm{n})$, equation for $e(\mathrm{n})$ is considered as follows:

$$
e(n)=d(n)-y(n)
$$

And for general cases, the next step is described by:

$$
W(n+1)=W(n)+2 \mu e(n) X(n)
$$

To minimize the error, the optimal value of $\mu$ is determined by empirical experiments. Based on the model, the next iteration is described by:

$$
\mu(n+1)=\left\{\begin{array}{l}
\mu(n) \rightarrow \Delta e^{2}(n)>0 \\
\mu(n) \times \alpha \rightarrow \beta<\Delta e^{2}(n)<0 \\
\mu(n) \times \alpha \rightarrow \beta<\Delta e^{2}(n)<0 \text { and } \operatorname{MRxe}(n) \leq 50 \times \operatorname{ARxe}(n) \\
\lambda_{\max } \rightarrow \beta<\Delta e^{2}(n)<0 \text { and } \operatorname{MRxe}(n)>50 \times \operatorname{ARxe}(n)
\end{array}\right.
$$

Where,

$$
\begin{aligned}
& \Delta e^{2}(n)=\left[e^{2}(n-1) e^{2}(n)\right] / e^{2}(n-1) \times 100 \\
& \operatorname{MRxe}(n)=\max \left(R_{x e(n)}(m)\right), \operatorname{ARxe}=\operatorname{mean}\left(R_{x e(n)}(m)\right) \\
& R_{x e(n)}(m)=\sum_{i=k-l+1}^{k} x(i) e(i)
\end{aligned}
$$

Bluetooth wireless transmission link is considered in this research. Bluetooth technique has many advantages which allows it to be suitable for this aim, namely the low-power consumption, miniature transmitter size and the low-cost. These signals are forwarded to the remote PC (server) to be processed for diagnosis. The complete architecture of this system is shown in Fig. 4.

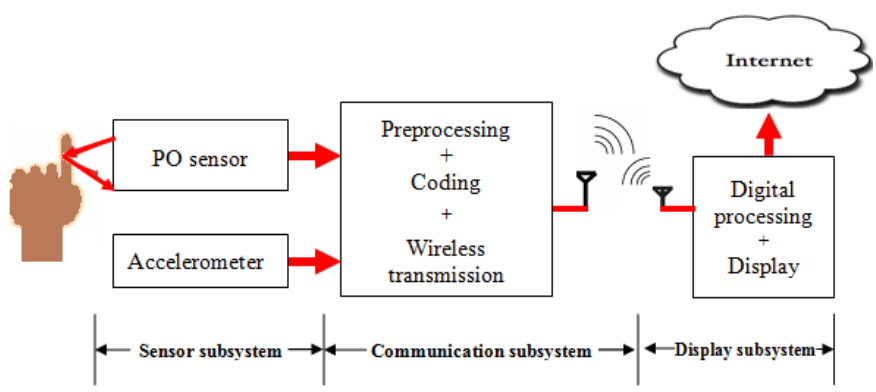

Fig. 4. Overall wearable PPG monitoring system architecture

\section{FINDINGS}

In this research, upon the investigation and experiments, the followings are found:

- The most suitable place for applying the PO to the human body in a wearable or portable form is on the finger. Thus, choice of sensor's shape is made for the finger-ring.

- The vital signals acquired from the heart, PPG, are subjected to a high motion level, due to the movement of the patient. Applying the developed VSS-ANC stabilizes the detected signals to an acceptable diagnostic level.

The results of the developed model and some other models are represented in Fig. 5.

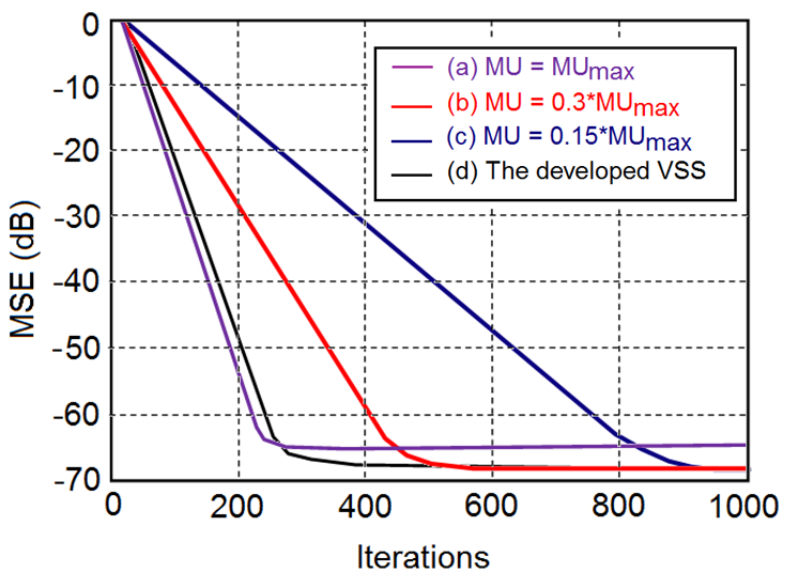

Fig. 5. The result of the developed VSS-ANC

Upon this algorithm, variety of acquired signals with additive noises are tested and it has been found that the acceptable output signal can be obtained from a total noisy environment of SNR $-35.4 \mathrm{~dB}$.

\section{CONCLUSION}

In conclusion, some of the critical issues related to the design of wearable PO sensor for telemedicine purposes are investigated and developed. The design is based on the fingerring shaped PO sensor, which can be worn anytime. Giving the patient movement freedom causes distortion in the acquired signals and thus, a robust VSS-ANC has been developed for reducing the motion artifact that ruins the desired signal. In the 
developed algorithm, it proves the superior performance of the output compared to the most common developed methods. Nevertheless, the algorithm has its own performance limit which is found to be acceptable up to SNR $-35.4 \mathrm{~dB}$ and it is generally acceptable for the wearable sensors.

\section{REFERENCES}

[1] S. Rhee, B. Yang, and H. Asada, "The ring sensor: a new ambulatory wearable sensor for twenty-four hour patient monitoring," IEEE Proc. Engineering in Medicine and Biology Society, vol. 20, pp. 1906-1909, 1998.

[2] N. Holter, "New method for heart studies: continuous electrocardiography of active subjects over long periods is now practical," Science, vol. 134, 1961, pp. 1214-1220.

[3] S. Jayaramam, and P. Sungmee, "Wearable sensor system: opportunities and challenges," IEEE Proc. pp.4153-4155.

[4] J. G. Webster, "Design of pulse oximeters," IOP Publishing, Bristol and Philadelphia, 1997.
[5] E. Zahedi, G. K. Beng, "Applicability of adaptive noise cancellation to fetal heart rate detection using photoplehtysmography," J. Comp. Biol. Med., vol. 38, pp. 31-41, Jan. 2008.

[6] T. Vo-Dinh, "Biomedical photonics handbook," CRC Press, 2003.

[7] L. I. Grossweiner, "The science of phototherapy: an introduction," Springer Publishing, Netherland, 2003.

[8] M. H. Niemz, "Laser-tissue interactions fundamentals and applications," $3^{\text {rd }}$ Enlarged Edition, Springer Publishing, 2007.

[9] Z. Karwiecki, A. Cysewska-Sobusiak, G. Wiczynski and A. Odon, "Modeling and measurements of light transmission through human tissues," Bull. Pol. Ac.: Tech., vol. 56, no. 2, pp. 147-154. 2008.

[10] K. K. Tremper, "Pulse oximetry," Chest, vol. 95, no. 4, pp. 713-715, April 1989.

[11] D. Bansal, M. Khan, A. Salhan, "Real time acquisition and pc to pc wireless transmission of human carotid pulse waveform," Computers in Biology and Medicine, vol. 39, no. 10, pp. 915-920, 2009. 